\title{
Hydrophobicity as a parameter to quantify relative efficacy of receptor determinants during host virus interaction
}

\section{Om Prakash*}

The Institute of Mathematical Sciences (IMSc), Chennai-600113, India

\begin{abstract}
The present study is an analytical description of a moment when surface protein of virus recognizes the surface part (receptor determinants) at host. Success of recognition is very first step of starting of viral infection. This momentarily derived mechanism has importance because it can be used for creating challenge for viral infection. But kinetics as well as components involved in this momentarily event is unexplored. Here study has been performed for identification of governing parameter as well as kinetics which can be used to quantify the relative efficiency of various receptor determinant analogues. The study was also exemplified for Corona virus to prioritize receptor determinant analogues. This protocol model can also be utilized as add-on for theoretical modeling of viral infectivity of host cell.
\end{abstract}

Keywords: Determinants; Efficacy; Hydrophobicity; Receptor; Virus.

*Corresponding Author:

\section{Om Prakash}

Post-Doctoral Fellow, Computational Biology

The Institute of Mathematical Sciences (IMSc), Chennai-600113, India

Email: mailbox4op@gmail.com,omprakash@imsc.res.in 


\section{INTRODUCTION}

Host virus interaction has been studied many times for their infection capacity as well as their multiplicity. As in case of infectious bronchitis Corona virus, correlation among the envelope proteins and $\mathrm{pH}$ was explained for extent of viral infection (Westerbeck and Machamer, 2019). In a study interaction between the newly designed inhibitors for Japanese encephalitis virus was evaluated for targeting protein envelope (Mormile and Vittori, 2014). In another study, interaction between the HIV virus and host cell membrane was explained in context of dependency on composition of cell membrane; where importance of hydrophobic properties for molecular interaction was also notified (Barros et al., 2016). Host-viral interaction was also found to be utilized for designing the inhibitors against NS1A protein of Influenza-A virus (Ai et al., 2014). At level of deep molecular study it was also observed that electrostatic properties and recruitment of Alpha receptor components are related with some viral interleukin-6. Here hydrophobic properties were notified during analysis (Perret et al., 2005).

Many attempts have been made to capture kinetic parameters for host-virus interaction. Kinetic parameters for virus growth have been explained for influenza-A virus in genetically engineered cell lines (Laske et al., 2019). Some of the kinetics analysis has been reported for influenza virus-receptor in binding analysis which was dependent on surface properties of receptors (Guo et al., 2018). Modeling studies were also known for infection of Flavivirus in a system of pluripotent stem cell (Shanko et al., 2019). Besides these, mouse models were also presented for Kinetic hematological analysis for Corona virus (Leist et al., 2019).

Corona viruses are one of the reasons for pneumonia worldwide. Its trans-membrane spike glycoprotein interacts with host receptors. It follows fast kinetics through surface-exposed groove at Spike-mediated viral entry into host cells. For influenza anti-viral activity, many mechanisms have been developed for barricading between virus and host cell. Out of these mechanisms, inhibition of interaction between host and virus is one of the concerned mechanisms. Such mechanisms of interaction require Receptor Determinants. Sialic Acid is known as Receptor Determinants for Corona viruses (Schwegmann-Weßels and Herrler, 2006). A Sialic Acid Analogue acts as a Receptor Determinant for Binding but not for infection (Brossmer et al., 1993). The receptor-interacting site is conserved in Spike glycoprotein for interaction with Sialoglycans at host cell surface. This conservation has been used for identification of receptor determinant analogues (Maurya, 2020). 
In general, Pharmacophore mapping is the concept used for generation of a profile for molecular interaction based on a set of small molecules against specific binding site. This process includes a set of small molecules with their 3D structure or residue orientation at binding site. Pharmacophore based screened molecules can be accessed as analogues acting at specific binding site. Considering all the above aspects, present study has been performed to define a customized kinetic model which can be used to quantify the relative efficacy of various receptor determinant analogues. The study has also been exemplified for corona virus to prioritize receptor determinant analogues extracted through ZINC database.

\section{MATERIAL \& METHOD}

Parameters for host-virus interaction: Hydrophobicity was considered as parameter to model host-virus interaction kinetics. Reason behind this step was to utilize information from real condition present over cell surface and extracellular environment where virus comes to interact with host. An average $\mathrm{pH}$ of the environment was considered as 7.0. Environment was considered as full of combination of hydrophilic and hydrophobic materials. Effect of $\mathrm{pH}$ was also included in study as the basis for distribution of charges during the molecule interaction. In whole system, ligand-receptor interaction involves a pocket of residues providing nested hydrophobic environment. Considering all these aspects $\log \mathrm{P}, \log \mathrm{D}, \mathrm{pH}$, and hydrophobicity were found to be possible parameters for the system of virus-host interaction.

Definition of host-virus interaction on the basis of hydrophobicity: Ligand derived hydrophobicity was used for model development. Reason behind this step was that no quantifying method is available for cumulative presentation of impact of hydrophobicity on attraction of ligand towards viral spike. Therefore it was assumed that the hydrophobicity calculated for ligand itself can be used for relative evaluation of receptor determinants as well as their analogues against single specific binding pocket of viral spike. Absolute value of difference between $\log P$ and $\log D$ was used as representative of maximum hydrophobicity by which ligand can be affected.

Kinetics model based on hydrophobicity: For a single binding site, it was pre-assumed that those ligands which are being evaluated should be derived from same pharmacophoric data-structure and should also be simulated within the same binding site. One of the important factors that have been considered is that each ligand spent some time during molecular interaction, and further showed their effect 
according to the maximum hydrophobicity value. These assumptions were found to be parametrically equivalent to the first derivative of 'Nelson-Siegel-Svensson' (NSS) model, where zero-rate has been observed at the maximum value of maturity parameter.

System of molecular interaction: System of molecular interaction contains two basic components including binding domain with residues and ligand. It was assumed that impact of hydrophobicity during ligand interaction will be represented into extent of closeness between receptor and ligand; and accordingly ligand will create impact on the behavior of receptor driving activity. This behavior can also be visualized as the extent of un-stability of the whole system after ligand interaction. Therefore the performance of ligand can also be evaluated on the basis of un-stability of the system of molecular interaction.

Case study for validation of model customization: Case study was performed with receptor determinant interaction with Corona virus. Receptor determinant which is recognized by a group of Corona viruses was used as reference; while analogues, derived on the basis of pharmacophore for binding site of receptor determinant were compared with reference receptor determinant and further model was evaluated and analyzed.

\section{RESULTS \& DISCUSSION}

The kinetic model was defined on the basis of 'Nelson-Siegel-Svensson' (NSS) model. This model was used to define Kinetics of interaction between receptor determinant and spike groove. This model was adopted to customize because of commonalities found between the skeleton of first derivative of NSS model and interaction between receptor determinant and spikes. It was observed that, when equally defined pharmacophore based small molecules get entered into the domain of binding site, their responses varies, which can also be used to define the performance of analogues as receptor determinants. Therefore the key components of the zero rate NSS model were restructured according to the requirement for molecular interaction. Ultimately ligand's ability to chase the viral spike was defined as the ratio of hydrophobicity and its impact on viral spike. Hydrophobicity based chasing capacity of ligand was defined by absolute value of difference of LogP and LogD; while impact of hydrophobicity was defined as the function of hydrophobicity and four more parameters $\alpha 1, \alpha 2, \alpha 3$ and $\beta$. Since it is the matter of interference between virus spike and human cell, therefore interfering molecule can interact with spike through which virus starts for entering into host. Assumptions have 
been made to select and customize the kinetics model for interaction of 'receptor determinant' vs. spike groove. It was assumed that testing molecule should be already qualified the pharmacophore requirement. When testing molecule will interacts with target i.e. spike groove of virus; it will ultimately affect the virus with different infectivity rate. Considering these assumptions, Nelson-Siegel-Svensson (NSS) model for forward rate was customized to define chasing ability of ligand towards spike of virus. This customized assumption can also be understood as: Since receptor determinant interacts with conserved pocket for some short of time. The duration of interaction is the function of extent of hydrophobicity. Therefore it was assumed that chasing efficacy can be defined by the capacity of interaction remained at the last moment of interaction defined by hydrophobicity. The last moment of interaction was defined by tenor point defined by extent of hydrophobicity. Therefore here kinetics rate was considered as integration of NSS forward rate up to the tenor point of maximum interaction.

Here Ligand is assumed to be a Receptor determinant analogue.

$$
\begin{gathered}
\text { Hydrophobicity chasing ability of ligand }(H)=\operatorname{abs}(\log P \sim \log D) \\
\text { Impact of } H \text { on Viral spike }(D)=f(H, \alpha 1, \alpha 2, \alpha 3, \beta) \\
D(H)=\alpha 1+(\alpha 2+\alpha 3) *(\beta / H) *(1-\exp (-H / \beta))-(a 3 * \exp (-H / \beta)) \\
\text { Ligand's ability to chase Viral Spike }=H / D(H)
\end{gathered}
$$

Where values for $\alpha 1, \alpha 2, \alpha 3, \beta$ will vary according to domain

Finally efficacy of ligand can be defined as:

$$
E=\frac{a b s(\log P \sim \log D)}{\left.\alpha 1+(\alpha 2+\alpha 3) *\left(\frac{\beta}{\operatorname{abs}(\log P \sim \log D}\right)\right) *\left(1-\exp \left(-\frac{\operatorname{abs}(\log P \sim \log D)}{\beta}\right)\right)-\left(a 3 * \exp \left(-\frac{\operatorname{abs}(\log P \sim \log D)}{\beta}\right)\right)}
$$

Zero rate was used for calibration of NSS model. The resultant was observed at the maximum hydrophobicity impact on the ligand, that's why the maturity parameter at NSS model was replaced by hydrophobicity parameter. Here points to be notified are that, in the present study extent of hydrophobicity was planned at $\mathrm{pH}$ equal to 7.0. NSS model equation derivative at tenor point represented a featured value for ligand protein interaction due to hydrophobicity of binding pocket. Binding site residues create hydrophobic involvement within the binding pocket where ligand has the accumulated possibilities to bind. But it cannot be quantified that what extent of hydrophobicity acts 
for attraction of ligand during molecular interaction. To overcome this problem, assumption of hydrophobicity value has been made on the basis of ligand itself. And it was also considered that when ligand protein interaction will be resulted due to impact of hydrophobicity of binding site then the maximum attraction which will be attain on ligand must be a function of hydrophobicity calculated for ligand. It was also assumed that extent of molecular interaction can also be represented by distance between the binding site and ligand. To present this parameter, distance between the centroids of binding site as well as ligand was considered. The NSS model derivative for zero rate was simulated for parameters governing relationship between hydrophobicity and the distance between the centroids. Here maturity parameter was replaced by hydrophobicity; because it was assumed that the calculated hydrophobicity value for ligand will represent the maximum attraction between ligand and protein. The simulated efficacy of ligands was found to follow NSS model distribution. Now to evaluate the reliability of customized model, a real ligand-receptor interaction was evaluated. Here receptor was binding pocket at glycoprotein spikes of a virus and ligand was receptor determinants available at cell surface of host. Here one natural occurring receptor determinant was used along with analogues derived from the pharmacophore of binding pocket at glycoprotein spike of virus. It was assumed that the naturally occurring receptor determinant should show highest efficacy over majority of the analogues, and if any analogue shows superior efficacy then receptor determinant may be a new possibility to prove competition with natural receptor determinant.

\section{Validation and Case Study}

Binding site domain, pharmacophore and identified receptor determinant analogues were considered from DOI: 10.26434/chemrxiv.12241634.v1; where study had been performed for identification of possible receptor determinant analogues for all 9-O-Ac-Sialoglycan-Recognizing Corona viruses. Here binding pocket contained two domains P1 \& P2. Present study adopted the initial dataset of protein and ligands from DOI: 10.26434/chemrxiv.12241634.v1. Both binding pockets were simulated, and found different parameter values of kinetics model (Table 1). Viral infectivity rate can be shown as direct impact of Ligand's ability to chase Viral Spike (Figure $1 \& 2$ ). List of 10 analogues and known receptor determinant (Supplementary Table S1) were processed through the kinetics model. Naturally known receptor determinant was found to highly efficient over majority of analogues identified through same pharmacophore. This case study validated the reliability of customized kinetic model.

Prioritization of receptor determinant analogues: Anti-corona virus receptor determinant analogues, identified in DOI: 10.26434/chemrxiv.12241634.v1, were evaluated through the system models of two 
domains of binding site. Phase plot comparison showed that ligands were creating un-stability in virus spike (Figure 3, 4, 5). Since two different domains were working, therefore no stable null point achieved. Analogue C3 was found to perform equivalent to receptor determinant. Therefore C3 can be used for anti-viral activity (Figure 6).

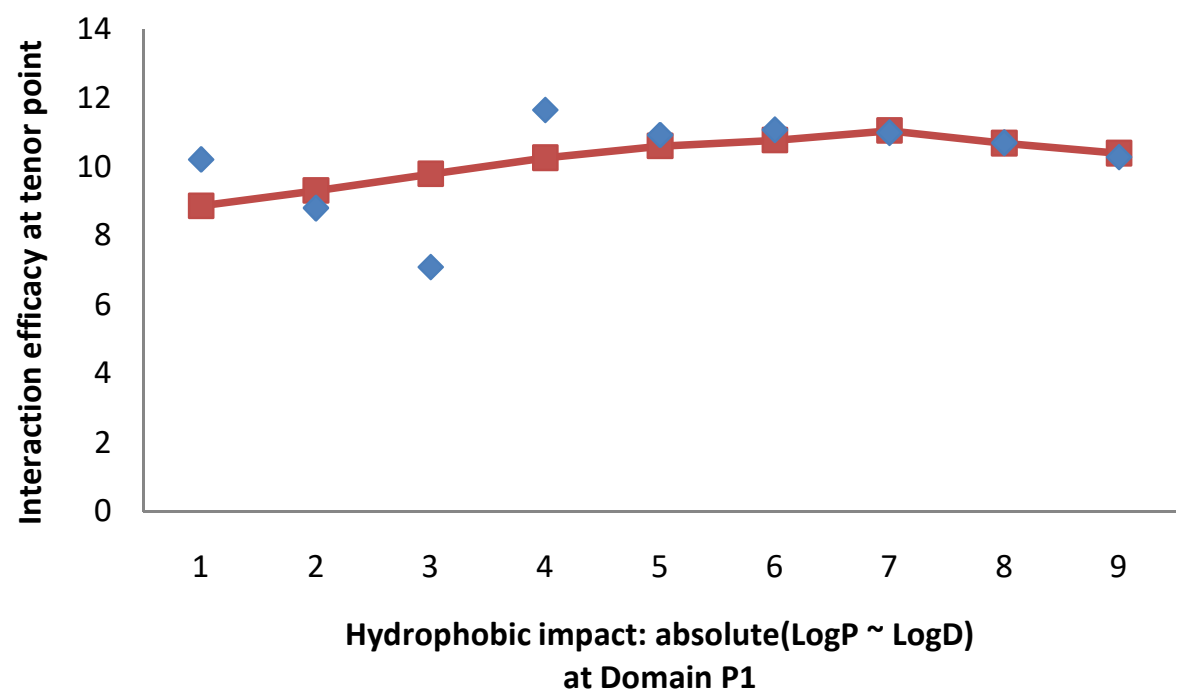

Figure 1. Viral infectivity rate can be shown as direct impact of Ligand's ability to chase Viral Spike at domain P1

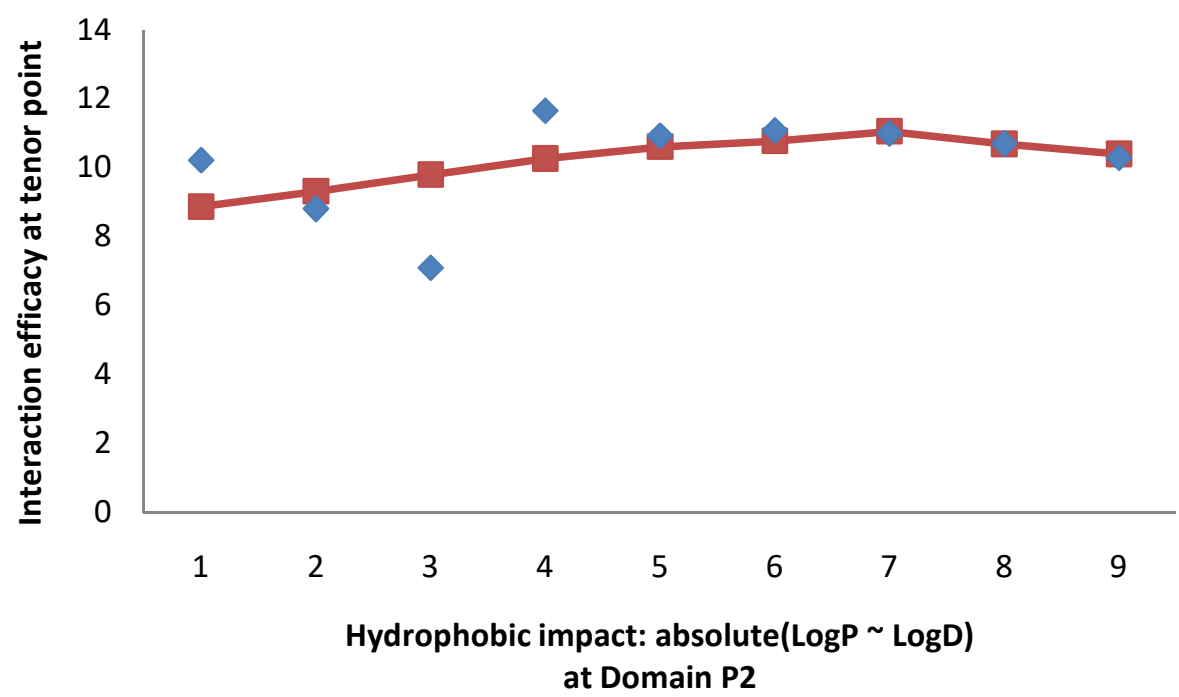


Figure 2. Viral infectivity rate can be shown as direct impact of Ligand's ability to chase Viral Spike at domain P2

Table 1. Model parameters optimized for two domains P1 \& P2

\begin{tabular}{|r|l|}
\hline Parameter & Domain P1 \\
\hline$\alpha 1$ & -465.707091 \\
\hline$\alpha 2$ & 474.1327822 \\
\hline$\alpha 3$ & 540.5571268 \\
\hline$\beta$ & 16.38474885 \\
\hline
\end{tabular}

\begin{tabular}{|r|l|}
\hline Parameter & Domain P2 \\
\hline$\alpha 1$ & -478.0579251 \\
\hline$\alpha 2$ & 484.9847989 \\
\hline$\alpha 3$ & 518.7311 .35 \\
\hline$\beta$ & 22.36179227 \\
\hline
\end{tabular}
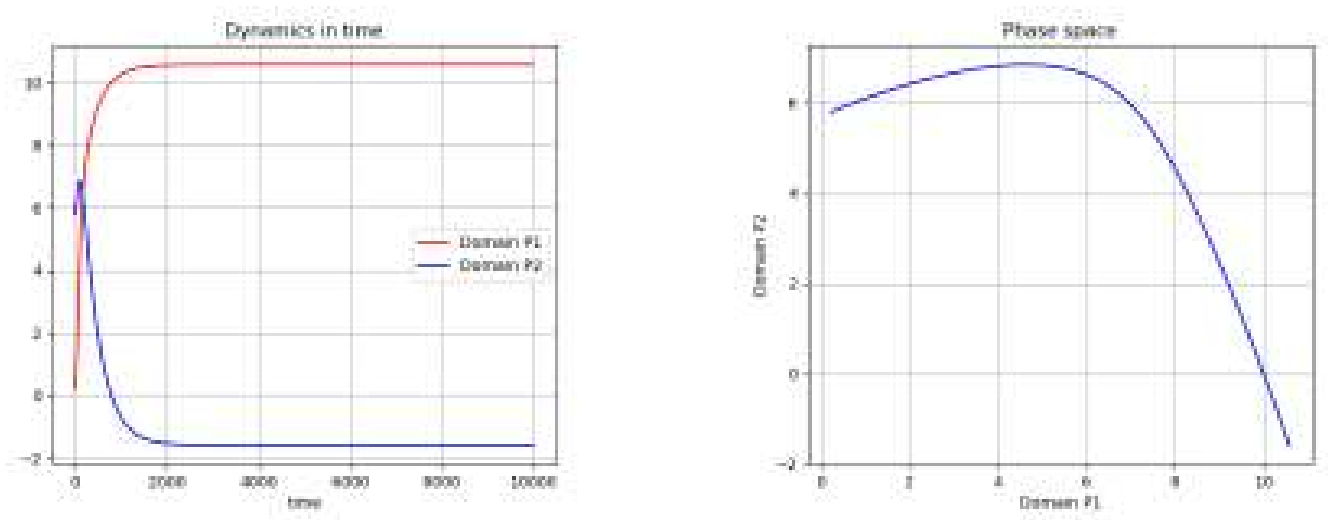

Figure 3. Generalized view of system of two domains P1 \& P2 interacting with receptor determinants
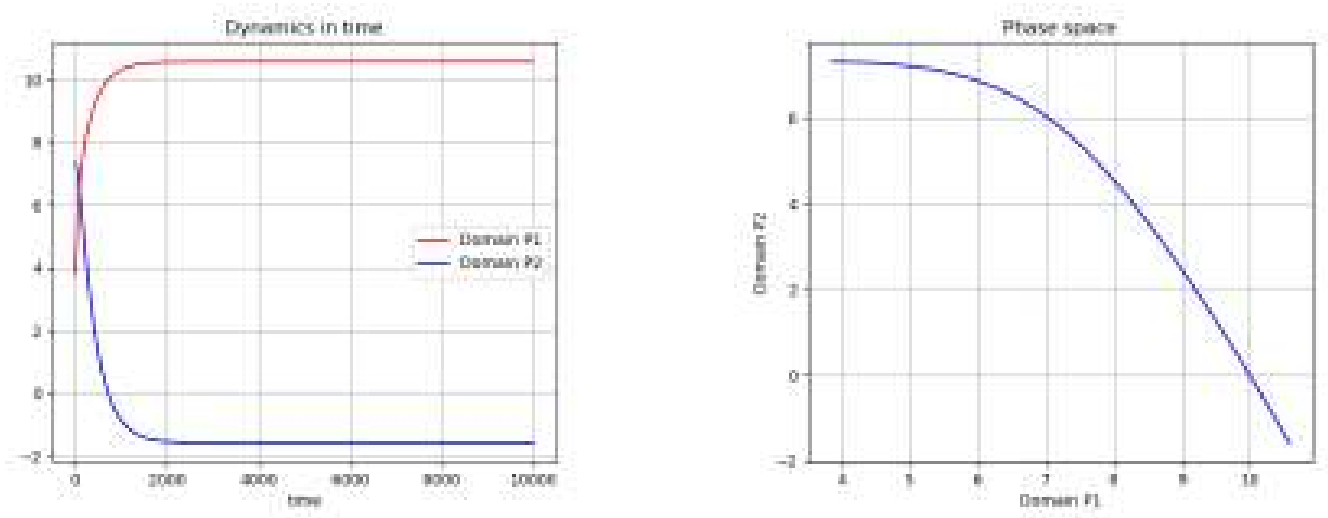

Figure 4. Co-Crystallized Ligand of 6NZK 

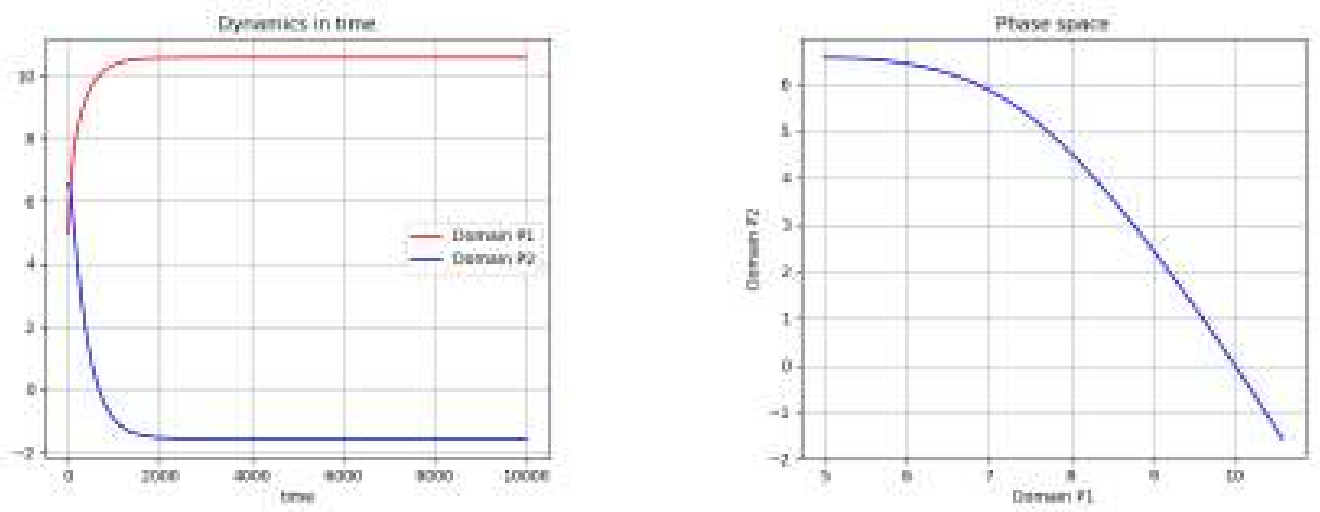

Figure 5. C3's performance similar to receptor determinant<smiles>CO[C@]1(C(=O)O)C[C@H](O)[C@@H](NC(C)=O)[C@H]([C@H](O)[C@H](O)COC(C)O)O1</smiles>

5-Acetylamino-6-[1,2-dihydroxy-3-(1-hydroxyethoxy)-propyl]-4-hydroxy-2-methoxy-tetrahydropyran-2-carboxylic acid

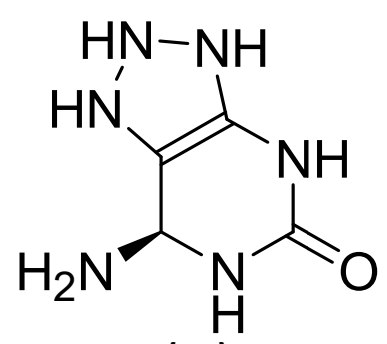

(C3)

Figure 6. C3 as prioritized analogue similar to natural receptor determinant

\section{CONCLUSION}

In the present study, ligand hydrophobicity was used to simulate kinetics of virus host interaction to quantify the relative efficacy of various receptor determinant analogues. Kinetic Model was proposed for viral-spike chasing capacity of Receptor determinant analogues. One analogue C3 was prioritized for anti-Corona virus activity, similar to the natural receptor determinant.

\section{ACKNOWLEDGEMENT}

Author expresses gratitude to The Institute of Mathematical Sciences, Chennai-600113, India for providing research facilities as well as DAE Post-Doctoral Fellowship (PDF 214). 


\section{REFERENCES}

Ai, H., Zhang, L., Chang, A.K., Wei, H., Che, Y., Liu, H., 2014. Virtual screening of potential inhibitors from TCM for the CPSF30 binding site on the NS1A protein of influenza a virus. J. Mol. Model. 20. https://doi.org/10.1007/s00894-014-2142-7

Barros, M., Heinrich, F., Datta, S.A.K., Rein, A., Karageorgos, I., Nanda, H., Lösche, M., 2016. Membrane Binding of HIV-1 Matrix Protein: Dependence on Bilayer Composition and Protein Lipidation. J. Virol. 90, 4544-4555. https://doi.org/10.1128/jvi.02820-15

Brossmer, R., Isecke, R., Herrler, G., 1993. A sialic acid analogue acting as a receptor determinant for binding but not for infection by influenza C virus. FEBS Lett. 323. https://doi.org/10.1016/00145793(93)81456-A

Guo, H., Rabouw, H., Slomp, A., Dai, M., van der Vegt, F., van Lent, J.W.M., McBride, R., Paulson, J.C., de Groot, R.J., van Kuppeveld, F.J.M., de Vries, E., de Haan, C.A.M., 2018. Kinetic analysis of the influenza $A$ virus HA/NA balance reveals contribution of NA to virus-receptor binding and NAdependent rolling on receptor-containing surfaces. PLoS Pathog. 14. https://doi.org/10.1371/journal.ppat.1007233

Laske, T., Bachmann, M., Dostert, M., Karlas, A., Wirth, D., Frensing, T., Meyer, T.F., Hauser, H., Reichl, U., 2019. Model-based analysis of influenza A virus replication in genetically engineered cell lines elucidates the impact of host cell factors on key kinetic parameters of virus growth. PLoS Comput. Biol. 15. https://doi.org/10.1371/journal.pcbi.1006944

Leist, S.R., Jensen, K.L., Baric, R.S., Sheahan, T.P., 2019. Increasing the translation of mouse models of MERS coronavirus pathogenesis through kinetic hematological analysis. PLoS One 14. https://doi.org/10.1371/journal.pone.0220126

Maurya, P.P., 2020. Receptor Determinant Analogues for All 9-O-Ac-Sialoglycan-Recognizing Corona Viruses. https://doi.org/10.26434/CHEMRXIV.12241634.V1

Mormile, R., Vittori, G., 2014. Endometriosis and susceptibility to tuberculosis: Is interferon- $\gamma$ the critical player? Eur. J. Obstet. Gynecol. Reprod. Biol. https://doi.org/10.1016/j.ejogrb.2013.12.040

Perret, D., Rousseau, F., Tran, V., Gascan, H., 2005. Reversal of some viral IL-6 electrostatic properties compared to IL- 6 contributes to a loss of alpha receptor component recruitment. Proteins Struct. Funct. Genet. 60, 14-26. https://doi.org/10.1002/prot.20445

Schwegmann-Weßels, C., Herrler, G., 2006. Sialic acids as receptor determinants for coronaviruses. Glycoconj. J. https://doi.org/10.1007/s10719-006-5437-9

Shanko, E.S., van de Burgt, Y., Anderson, P.D., den Toonder, J.M.J., 2019. Microfluidic magnetic mixing at low reynolds numbers and in stagnant fluids. Micromachines 10.

https://doi.org/10.3390/mi10110731

Westerbeck, J.W., Machamer, C.E., 2019. The Infectious Bronchitis Coronavirus Envelope Protein Alters Golgi pH To Protect the Spike Protein and Promote the Release of Infectious Virus. J. Virol. 93. https://doi.org/10.1128/jvi.00015-19 


\section{Supplementary Table}

Table S1. Natural receptor determinant and 10 analogues with possibility to be identified with Corona virus (Reference: DOI: 10.26434/chemrxiv.12241634.v1)<smiles>CO[C@]1(C(=O)O)C[C@@H](O)[C@H](NC(C)=O)[C@H]([C@H](O)[C@@H](O)COC(C)O)O1</smiles>

5-Acetylamino-6-[1,2-dihydroxy-3(1-hydroxy-ethoxy)-propyl]-4hydroxy-2-methoxy-tetrahydropyran-2-carboxylic acid<smiles>CNC(=O)NC(=O)N1CC=CN1</smiles>

(C2)<smiles>O=c1[nH]c2c(c(=O)[nH]1)NSN2</smiles>

(C5)<smiles>CC(C)(C)C1=C[C@@H]2NC(N)NC(N)N2N1</smiles>

(C8)<smiles>N[C@@H]1NC(=O)NC2=C1NNN2</smiles>

(C3)<smiles>O=C1NC(=O)C2NCN(C(=O)O)C2N1</smiles>

(C6)<smiles>O=c1[nH]c2c(c(=O)[nH]1)OC(c1ccccc1)N2</smiles>

(C9)<smiles>C[C@@H]1NC2=C(NON2)NC1=O</smiles>

(C1)<smiles>CC1=CC2NC(N)NC(N)N2N1</smiles>

(C4)<smiles>O=c1[nH]c(=O)c2oc3c(c2[nH]1)C=CCN3</smiles>

(C7)<smiles></smiles> 